Schriften zur Reform des Verwaltungsrechts

Wolfgang Hoffmann-Riem / Eberhard Schmidt-Aßmann (Hrsg.)

Verwaltungsverfahren und

Verwaltungsverfahrensgesetz

Nomos Verlagsgesellschaft

Baden-Baden 
Schriften zur Reform des Verwaltungsrechts

Herausgegeben von:

Prof. Dr. Wolfgang Hoffmann-Riem

Prof. Dr. Eberhard Schmidt-Aßmann

Band 9 
Prof. Dr. Wolfgang Hoffmann-Riem /

Prof. Dr. Eberhard Schmidt-Aßmann (Hrsg.)

\section{Verwaltungsverfahren und Verwaltungsverfahrensgesetz}

Nomos Verlagsgesellschaft Baden-Baden 
Gedruckt mit Unterstützung der Deutschen Forschungsgemeinschaft.

Bibliografische Information Der Deutschen Bibliothek

Die Deutsche Bibliothek verzeichnet diese Publikation in der Deutschen Nationalbibliografie; detaillierte bibliografische Daten sind im Internet über http://dnb.ddb.de abrufbar.

ISBN 3-7890-8040-3

1. Auflage 2002

(c) Nomos Verlagsgesellschaft, Baden-Baden 2002. Printed in Germany. Alle Rechte, auch die des Nachdrucks von Auszügen, der photomechanischen Wiedergabe und der Übersetzung, vorbehalten. Gedruckt auf alterungsbeständigem Papier. 


\section{Vorwort der Herausgeber}

Verwaltungsverfahren sind planvoll geordnete Vorgänge zur Gewinnung und Verarbeitung von Informationen bei der Bewältigung öffentlicher Aufgaben. Das Verfahren erlaubt es allen Beteiligten, die jeweils eigenen Interessen und damit auch die eigene Sichtweise in den Problemlösungsprozess einzubringen. Insofern dienen Verfahren der Interessenklärung und damit zugleich der Verbesserung der Rationalität administrativen Handelns. Zahlreiche Verfahren zielen zudem auf einen dem Gerichtsverfahren vorgelagerten Rechtsschutz. Je weniger das administrative Verhalten durch exakt formulierte Gesetzesprogramme vorgezeichnet ist, je mehr Beurteilungs- und Gestaltungsspielräume die Verwaltung also vorfindet, desto wichtiger wird die Aufgabe des Verfahrens, durch Erfassung der vielfältigen Fakten und der maßgebenden Werte unter Einschluss der individuellen Interessen der Akteure eine gute Grundlage für die Erarbeitung eines Entscheidungsergebnisses zu finden, das nicht nur rechtliche Vorgaben wahrt, sondern im Rahmen des Zugelassenen auch Optimierungen vornimmt und dadurch Akzeptanz ermöglicht. Die neuen informationstechnischen Gegebenheiten schaffen dabei neue Möglichkeiten der Vernetzung und damit auch der Gleichartigkeit der Verfahrensteilhabe vieler.

Richtigkeitsgewähr durch Verfahren wird zu einer eigenständigen Funktion. Eine solche nicht nur am Rechtsschutz orientierte Sichtweise hat durch die Europäisierung der Rechtsordnung, auch des Verwaltungsrechts, Auftrieb erhalten. Auf europäischer Ebene werden gegenwärtig Konzepte "guter administrativer Praxis « (good governance) forciert, die an Grundsätzen der Offenheit, Partizipation, Verantwortlichkeit, Effektivität und Kohärenz orientiert sein sollen. Ihre Einzelausprägungen sind nur begrenzt durch Recht vorgegeben, sie wirken aber auf die Verwirklichung rechtlicher Ziele in administrativen Verfahren ein.

Demgegenüber ist das im deutschen Verwaltungsverfahrensgesetz verankerte Verfahrenskonzept gegenwärtig noch enger, vorrangig am Typ gesetzesvollziehender Verwaltung orientiert. Zum Teil neuartige Verfahrenskonzepte finden sich allerdings in vielen bereichsspezifischen Sondergesetzen (etwa im Umwelt-, Informations-, Telekommunikations- und Produktsicherungsrecht). Darüber hinaus hat die Praxis eigene Formen der Informationsverarbeitung, insbesondere im Zuge kooperativer Problembewältigung, entwickelt und dabei die im Verfahrensrecht enthaltenen Flexibilitätsmargen ausgiebig genutzt. Damit aber drohen die »alten Ziele« der Kodifikation des Verfahrensrechts geschwächt zu werden, nämlich die der Vereinheitlichung, der Übersichtlichkeit und Rechtssicherheit, aber auch der Vereinfachung. 
Neue Herausforderungen im europäischen und deutschen Recht, neue Verfahrenskonzepte und neue Typen von Verwaltungsentscheidungen (etwa Versteigerungsverfahren oder Qualitätssicherungsverfahren) provozieren die Frage nach dem Reformbedarf des Verfahrensrechts. Die in diesem Band enthaltenen Beiträge diskutieren diese Frage vor dem Hintergrund der vor nunmehr zehn Jahren begonnenen, in den "Schriften zur Reform des Verwaltungsrechts« dokumentierten Diskussionen um eine Modernisierung des Verwaltungsrechts. Den Beiträgen ist ein DFG-Rundgespräch vorangegangen, das im November 2001 in Hamburg stattgefunden hat. Die Aufsätze nehmen das Verfahrensrecht als Ordnungsidee, um seine Prämissen und Erscheinungsformen vor dem Hintergrund längerfristiger Entwicklungslinien und aktueller Änderungsbedarfe zu reflektieren: Zu erfassen sind intra- und interbehördliche Informations-, Klärungs- und Aufsichtsverfahren; in den Blick geraten informelle Absprachen im Vorfeld oder in Begleitung von formellen Verfahren; einzuordnen sind Verfahren realer Leistungsbewirkung, Planungsverfahren, Zulassungsverfahren (Eröffnungskontrollen u. ä.), Auswahl- und Verteilungsverfahren (wie Vergabe- und Versteigerungsverfahren), Verfahren laufender Überwachung (z. B. Risikoaufsicht), aber auch Verfahren zur hoheitlichen Wahrnehmung der Gewährleistungs- und Auffangverantwortung des Staates im Bereich gesellschaftlicher Selbstregulierung (etwa Zertifizierung, Akkreditierung, Auditierung). Zu analysieren sind unterschiedliche Verfahrensschritte und deren Vernetzung sowie das Zusammenspiel von Förmlichkeit und Nichtförmlichkeit. Zu berücksichtigen sind Machtasymmetrien in der Verfahrensteilhabe, etwa bei der Informationsaufnahme oder dem Zugriff auf Expertenwissen. Immer wichtiger werden die internationalen, insbesondere europarechtlichen Impulse. Verfahren der europäischen Verwaltungskooperation sind ebenso zu behandeln wie die europabedingte Umorientierung nationalen Rechts. Die europäische Dimension wird dementsprechend in die Einzelanalysen einbezogen, also nicht in ausgegliederter Weise thematisiert.

Die Beiträge arbeiten neue Verfahrenselemente heraus, fragen nach ihrer Verallgemeinerungsfähigkeit und wollen die vielfältigen Entwicklungen in Ordnungskonzepte eingliedern. Sie analysieren das Spannungsverhältnis zwischen evolutionären Änderungen in der Rechtsdogmatik und der Kodifikation neuer Verfahrensformen und -typen. Für einzelne Bereiche wird Kodifikationsbedarf herausgearbeitet, nicht aber die Forderung nach einer Gesamtkodifikation des Verwaltungsverfahrensrechts erhoben. Die Beschäftigung mit dem Verfahrensrecht ist ein wichtiges Anwendungsfeld für das Bemühen, die Reform des Verwaltungsrechts im Rahmen des Möglichen auch durch die problemangemessene Auslegung und Anwendung vorhandenen Rechts und die Modifikation überkommener rechtsdogmatischer Figuren voranzutreiben. 


\section{Inhaltsverzeichnis}

Wolfgang Hoffmann-Riem

Verwaltungsverfahren und Verwaltungsverfahrensgesetz -

Einleitende Problemskizze

Wolfgang Kahl

Das Verwaltungsverfahrensgesetz zwischen Kodifikationsidee

und Sonderrechtsentwicklungen

Heribert Schmitz

Fortentwicklung des Verwaltungsverfahrensgesetzes:

Konkrete Gesetzgebungspläne und weitere Perspektiven

Martin Burgi

Die Funktion des Verfahrensrechts in privatisierten Bereichen

- Verfahren als Gegenstand der Regulierung nach Verantwortungsteilung

Michael Holoubek

Die Bedeutung des Untersuchungsgrundsatzes im Verwaltungs-

verfahren

Gabriele Britz

Reaktionen des Verwaltungsverfahrensrechts auf die informationstechnischen Vernetzungen der Verwaltung

Andreas Voßkuhle

Strukturen und Bauformen ausgewählter neuer Verfahren

Jan Ziekow

Der Einfluss des neuen Steuerungsmodells auf das Verwaltungsverfahren und seine gesetzliche Regelung

Ulrich Ramsauer

Das Verwaltungsverfahrensrecht im Spiegel der Kommentarliteratur 
Michael Gerhardt

Funktionaler Zusammenhang oder Zusammenstoß zweier Rationalitäten?

Das Verhältnis von Verwaltungsverfahren und Verwaltungsprozess am Beispiel der jüngeren Verfahrensfehlerlehre

Eberhard Schmidt-Aßmann

Verwaltungsverfahren und Verwaltungsverfahrensgesetz -

Perspektiven der Systembildung 